05

\title{
Тепловые механизмы необратимого разрушения сверхпроводящих свойств технических сверхпроводников
}

\author{
(C) В.P. Романовский \\ Национальный исследовательский центр „Курчатовский институт“, \\ 123182 Москва, Россия \\ e-mail: romanovskii@aol.com
}

(Поступило в Редакцию 25 октября 2016 г.)

\begin{abstract}
Сформулированы особенности необратимого распространения тепловых неустойчивостей в технических сверхпроводниках при различных условиях их охлаждения (интенсивном и неинтенсивном). Анализ проведен с использованием двух моделей вольт-амперной характеристики сверхпроводника: идеальной, предполагающей скачкообразный переход из сверхпроводящего состояния в нормальное, и непрерывной, описываемой степенным уравнением. Вычислены скорости распространения тепловых неустойчивостей вдоль композита. Расчеты выполнены как для докритических, так и для закритических токов. Показано, что они распространяются вдоль технического сверхпроводника в виде волны переключения. При этом у интенсивно охлаждаемых технических сверхпроводников во всем диапазоне изменения тока установившиеся значения скорости необратимого распространения теплового возмущения в продольном направлении могут быть только положительными в силу отсутствия области стационарной стабилизации. Доказано, что по своему характеру развития процесс нарастания температуры технического сверхпроводника при необратимом распространении тепловой неустойчивости аналогичен диффузионным явлениям, которые протекают при цепных реакциях взрывного типа.
\end{abstract}

DOI: $10.21883 /$ JTF.2017.08.44725.2080

\section{Введение}

Изучение теплоэлектродинамических явлений, происходящих в сверхпроводящих средах, лежит в основе решения многих проблем физики сверхпроводников, которые в макроскопическом приближении позволяют найти границу стабильных сверхпроводящих состояний при действии возмущений различной природы. Значительную роль в понимании принципов сохранения работоспособности технических сверхпроводников играет теория тепловой стабилизации [1-3]. В ее рамках для описания происходящих в них явлений широко используется идеальная вольт-амперная характеристика (BAX) сверхпроводника, предполагающая скачкообразный переход из сверхпроводящего состояния в нормальное. В результате диапазон рабочих токовых состояний технических сверхпроводников ограничивается критическим током. Однако как низкотемпературные, так и высокотемпературные сверхпроводники имеют непрерывно нарастающие BAX, которые наблюдаются в широком диапазоне изменения напряженности электрического поля. Их учет позволил объяснить ряд явлений, происходящих в композитных проводниках на основе низкотемпературных и высокотемпературных сверхпроводников, и которые не могут быть объяснены в рамках модели с идеальной BAX (см., например, [4-8]). В связи с этим в настоящей работе обсуждаются особенности теплового разрушения сверхпроводящих свойств композита, изготовленного из высокотемпературного сверхпроводника со степенным уравнением ВАХ, широко используемым для описания реального перехода из сверхпроводящего состояния в нормальное. При этом во внимание были приняты нелинейные температурные зависимости не только критической плотности сверхпроводника, характерные для высокотемпературных сверхпроводников в силу гигантского крипа магнитного потока, но и теплоэлектрофизических свойств стабилизирующей матрицы.

\section{Постановка задачи}

Исследуем разогрев тонкого композитного проводника $(-l<x<l)$ с площадью поперечного сечения $S$, состоящего из высокотемпературного сверхпроводника в несверхпроводящей матрице с удельным электрическим сопротивлением $\rho_{m}$, в результате действия конечного температурного возмущения, когда он мгновенно нагрелся до температуры $T_{1}$ на участке $-x_{1}<x<x_{1}$. Предположим, что композит с периметром охлаждения $p$ находится в постоянном магнитном поле $B$ и по нему течет постоянный ток с плотностью $J$ в направлении продольной оси $X$; температура $T$ и напряженность электрического поля $E$ по сечению композита не меняются; между сверхпроводником и матрицей имеются идеальные тепловой и электрический контакты; ВАХ сверхпроводника описывается либо моделью, предполагающей скачкообразный переход из сверхпроводящего состояния в нормальное, либо степенным уравнением. Тогда изменение температуры во времени и по длине композита $\left(t>0,-l<x<l, l \ll x_{1}\right)$, с боковой поверхности которого в хладагент с температурой $T_{0}$ отводится тепловой поток $q(T)$, может быть определено 
из решения следующего одномерного уравнения теплопроводности:

$$
C(T) \frac{\partial T}{\partial t}=\frac{\partial}{\partial x}\left[\lambda(T) \frac{\partial T}{\partial x}\right]-q(T) \frac{p}{S}+E J
$$

с начально-краевыми условиями

$$
\begin{gathered}
T(x, 0)=\left\{\begin{array}{ll}
T_{1}=\mathrm{const}, & |x| \leq x_{1} \\
T_{0}=\mathrm{const}, & x_{1}<|x|<l
\end{array},\right. \\
\frac{\partial T}{\partial x}(0, t)=0, \quad T(l, t)=T_{0},
\end{gathered}
$$

учитывающими симметричное относительно начала координат изменение температуры композита.

Для сверхпроводника со скачкообразной BAX напряженность электрического поля при линейной температурной зависимости критической плотности тока сверхпроводника вида $J_{c}=J_{c 0}\left(T_{c B}-T\right) /\left(T_{c B}-T_{0}\right)$ описывается формулой [1-3]

$$
\begin{aligned}
& E(T)=\frac{J \rho_{m}(T, B)}{1-\eta} \\
& \times\left\{\begin{array}{lc}
1, & T>T_{c B}, \\
\left(T-T_{c B}\right) /\left(T_{c B}-T_{c S}\right), & T_{c s} \leq T \leq T_{c B}, \\
0, & T<T_{c s}=T_{c B}-\left(T_{c B}-T_{0}\right) J /\left(\eta J_{c 0}\right) .
\end{array}\right.
\end{aligned}
$$

Здесь $J_{c 0}$ и $T_{c B}-$ критическая плотность тока сверхпроводника при температуре хладагента $T_{0}$ и его критическая температура для заданного внешнего магнитного поля соответственно, $T_{c s}$ - температура резистивного перехода, при которой транспортный ток равен критическому току композита, $\eta$ - коэффициент заполнения композита сверхпроводником.

Напряженность электрического поля для сверхпроводника со степенной ВАХ при заданных значениях плотности тока $J$ и температуры $T$ является решением системы уравнений

$$
\begin{gathered}
E=E_{c}\left[J_{s c} / J_{c}(T, B)\right]^{n}=J_{m} \rho_{m}(T, B), \\
J=\eta J_{s c}+(1-\eta) J_{m},
\end{gathered}
$$

где $J_{s c}$ и $J_{m}-$ плотности токов, протекающих в сверхпроводящей части композита и в стабилизирующей матрице соответственно, $J_{c}(T, B)$ - критическая плотность тока сверхпроводника, определенная на основе использования априори заданного значения напряженности электрического поля $E_{c}, n-$ параметр нарастания BAX.

Анализ условий тепловой стабилизации выполним для сверхпроводящего композита на основе $\mathrm{Bi}_{2} \mathrm{Sr}_{2} \mathrm{CaCu}_{2} \mathrm{O}_{8}$ в серебряной матрице и находящегося в магнитном поле с индукцией $B=15 \mathrm{~T}$, используя данные, приведенные в [9]. Соответственно представленные ниже результаты были получены на основе численного решения задачи $(1)-(4)$, выполненного при $l=50 \mathrm{~cm}, p=0.47 \mathrm{~cm}$,
$S=1.23 \cdot 10^{-2} \mathrm{~cm}^{2}, n=10, \eta=0.263, E_{c}=10^{-6} \mathrm{~V} / \mathrm{cm}$. Критическая плотность тока для сверхпроводника со степенной ВАХ рассчитывалась по формуле

$$
\begin{aligned}
J_{c}(T, B)= & J_{0}\left(1-\frac{T}{T_{c}}\right)^{\gamma}\left[(1-\chi) \frac{B_{0}}{B_{0}+B}\right. \\
& \left.+\chi \exp \left(-\frac{\beta B}{B_{c 0} \exp \left(-\alpha T / T_{c}\right)}\right)\right]
\end{aligned}
$$

при $T_{c}=87.1 \mathrm{~K}, \alpha=10.3, \beta=3.3, \gamma=1.73, \chi=0.27$, $B_{c 0}=465 \mathrm{~T}, \quad B_{0}=75 \cdot 10^{-3} \mathrm{~T}, \quad J_{0}=1.1 \cdot 10^{6} \mathrm{~A} / \mathrm{cm}^{2} \quad$ coгласно $[9,10]$.

Формула (5) использовалась для оценки значений $J_{c 0}$ и $T_{c B}$. При $B=15$ Т линейная аппроксимация приводит к следующим значениям: $J_{c 0}=1.736 \cdot 10^{5} \mathrm{~A} / \mathrm{cm}^{2}$, $T_{c B}=26 \mathrm{~K}$. В этом случае критический ток композита $I_{c}=\eta J_{c 0} S$ равен $561 \mathrm{~A}$ при $T_{0}=4.2 \mathrm{~K}$ и $I_{c}=154 \mathrm{~A}$ при $T_{0}=20 \mathrm{~K}$.

Для расчета удельного электрического сопротивления серебра использовались результаты $[11,12]$, принимая $\rho_{m}(273 K)=1.48 \cdot 10^{-6} \Omega \cdot \mathrm{cm}$ при задании $\mathrm{RRR}=$ $=\rho_{m}(273 \mathrm{~K}) / \rho_{m}(4.2 \mathrm{~K})$. Значение RRR полагалось равным 10.

В уравнении (1) объемная теплоемкость композита определялась как $C(T)=\eta C_{s}(T)+(1-\eta) C_{m}(T)$ с учетом теплоемкости сверхпроводника $C_{s}$ и матрицы $C_{m}$. Здесь температурная зависимость теплоемкости $\mathrm{Bi}_{2} \mathrm{Sr}_{2} \mathrm{CaCu}_{2} \mathrm{O}_{8}$ рассчитывалась по формуле

$C_{s}=\left\{\begin{array}{l}5.85 \cdot 10^{-5} T+2.2 \cdot 10^{-5} T^{3}, T \leq 10 \mathrm{~K} \\ -10.54 \cdot 10^{-2}+1.28 \cdot 10^{-2} T, T>10 \mathrm{~K}\end{array} \quad \mathrm{~J} /\left(\mathrm{cm}^{3} \mathrm{~K}\right)\right.$,

а объемная теплоемкость серебра $C_{m}$ была вычислена согласно [11]. Коэффициент теплопроводности для рассматриваемого композита рассчитывался по формуле

$$
\lambda(T)=\eta \lambda_{s}(T)+(1-\eta) 2.45 \cdot 10^{-8} T / \rho_{m}(T, B),
$$

учитывая закон Видемана-Франца. Согласно [13], коэффициент теплопроводности $\mathrm{Bi}_{2} \mathrm{Sr}_{2} \mathrm{CaCu}_{2} \mathrm{O}_{8}$ аппроксимировался следующим выражением:

$$
\begin{aligned}
& \lambda_{c}(T)[\mathrm{W} /(\mathrm{cm} \cdot \mathrm{K})]=\left(-1.234 \cdot 10^{-5}+1.654 \cdot 10^{-4} T\right. \\
& \left.+4.608 \cdot 10^{-6} T^{2}-1.27 \cdot 10^{-7} T^{3}+6.061 \cdot 10^{-10} T^{-4}\right)
\end{aligned}
$$

При задании теплового потока с боковой поверхности композита были рассмотрены различные режимы его охлаждения. Во-первых, предполагалось, что в качестве охладителя используются жидкие гелий или водород. В этих случаях температурная зависимость $q(T)$ характеризуется скачкообразным переходом [1] от пузырькового режима кипения к пленочному после его перегрева на величину $\Delta T_{\mathrm{cr}}$. Для данных хладагентов зависимость $q(T)$ при атмосферном давлении, учитывающая оба режима кипения, рассчитывалась согласно [14]. 
Соответственно для жидкого гелия она записывалась в виде

$$
q(T)\left[\mathrm{W} / \mathrm{cm}^{2}\right]= \begin{cases}2.15\left(T-T_{0}\right)^{1.5}, & T \leq T_{0}+\Delta T_{\mathrm{cr}} \\ 0.06\left(T-T_{0}\right)^{0.82}, & T>T_{0}+\Delta T_{\mathrm{cr}}\end{cases}
$$

при $T_{0}=4.2 \mathrm{~K}, \Delta T_{\mathrm{cr}}=0.6 \mathrm{~K}$ и

$$
q(T)\left[\mathrm{W} / \mathrm{cm}^{2}\right]= \begin{cases}0.6615\left(T-T_{0}\right)^{2.6}, & T \leq T_{0}+\Delta T_{\mathrm{cr}}, \\ 0.0024\left(T-T_{0}\right)^{1.1}, & T>T_{0}+\Delta T_{\mathrm{cr}}\end{cases}
$$

для жидкого водорода при $T_{0}=20 \mathrm{~K}, \Delta T_{\mathrm{cr}}=3 \mathrm{~K}$.

Во-вторых, тепловая стабильность $\mathrm{Ag} / \mathrm{Bi}_{2} \mathrm{Sr}_{2} \mathrm{CaCu}_{2} \mathrm{O}_{8}$ исследовалась для неинтенсивных условий охлаждения, которые возникают при охлаждении сверхпроводящего магнита криокулером, газообразным хладагентом, косвенном охлаждении токонесущих элементов за счет кондуктивного механизма перераспределения тепла между ними. В этом случае тепловой поток с поверхности композита в хладагент может быть рассчитан по формуле

$$
q(T)\left[\mathrm{W} / \mathrm{cm}^{2}\right]=h\left(T-T_{0}\right) .
$$

Она использовалась для анализа тепловых состояний композита при $h=10^{-3} \mathrm{~W} /\left(\mathrm{cm}^{2} \cdot \mathrm{K}\right)$, когда температура хладагента задавалась равной $T_{0}=4.2 \mathrm{~K}$ или $T_{0}=20 \mathrm{~K}$.

\section{Кинетика температурных возмущений при докритических и закритических токах}

Обсудим в рамках сформулированного нелинейного приближения характерные особенности необратимого

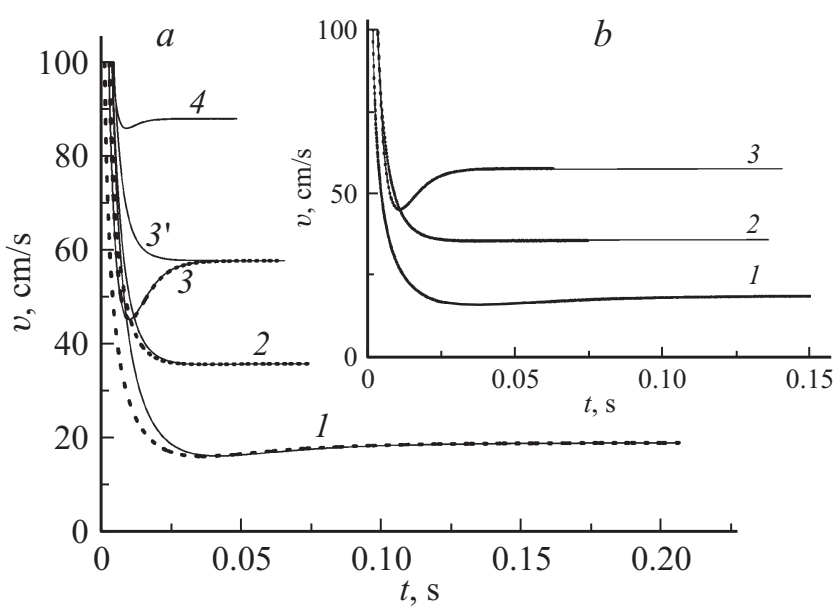

Рис. 1. Изменение во времени скорости тепловой неустойчивости $\left(a-\right.$ расчет по скорости распространения изотерм $T_{c s}$ и $T_{0}+1 ; b-$ расчет по скорости распространения изотермы $T_{c s}$ и эквипотенциала $\left.E_{c}\right)$ в результате действия локального возмущения $\left(x_{1}=0.5 \mathrm{~cm}\right)$ с различной начальной температурой при охлаждении композита жидким гелием: $1-I=300 \mathrm{~A}$, $T_{1}=30 \mathrm{~K} ; 2-I=400 \mathrm{~A}, T_{1}=30 \mathrm{~K} ; 3-I=500 \mathrm{~A}, T_{1}=20 \mathrm{~K}$; $3^{\prime}-I=500 \mathrm{~A}, T_{1}=30 \mathrm{~K} ; 4-I=600 \mathrm{~A}, T_{1}=20 \mathrm{~K}$.

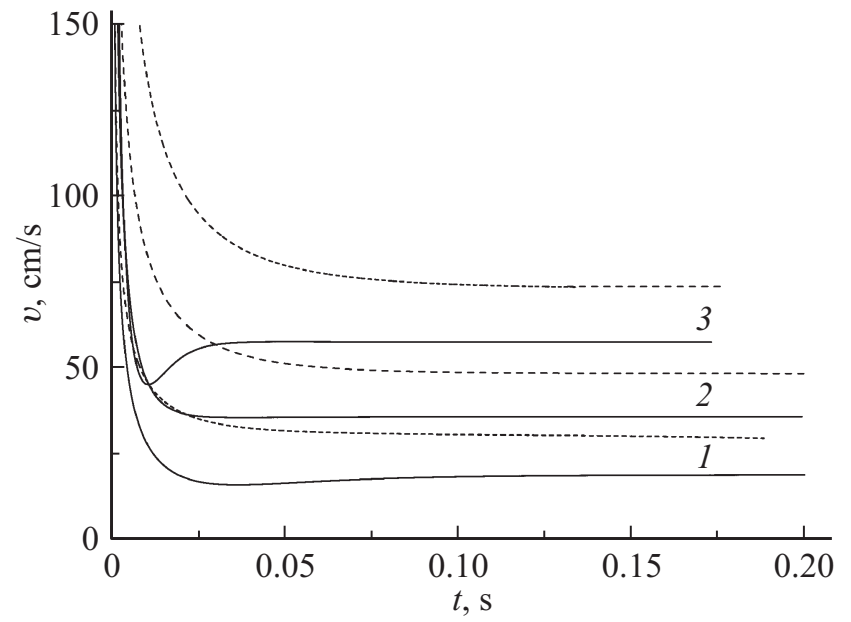

Рис. 2. Изменение во времени скорости изотермы $T_{c s}$ в результате действия локального возмущения $x_{1}=0.5 \mathrm{~cm}$, $T_{1}=30 \mathrm{~K}(1-I=300 \mathrm{~A}, 2-I=400 \mathrm{~A}, 3-I=500 \mathrm{~A})$ и различных условиях охлаждения: сплошная линия - жидкий гелий, штриховая линия - неинтенсивное охлаждение.

распространения тепловых возмущений вдоль композита. В терминах теории тепловой стабилизации в этом случае энергия начального возмущения должна превышать критическое значение [1-3]. Поэтому при проведении расчетов параметры начального возмущения $x_{1}$ и $T_{1}$ варьировались таким образом, чтобы тепловое возмущение было закритическим. Как будет показано ниже, правомерность их произвольного задания обусловлена тем, что при заданном транспортном токе тепловая неустойчивость после действия какого-либо закритического теплового возмущения по истечению переходного периода распространяется вдоль композита с одной и той же постоянной скоростью. При этом формируется так называемая волна переключения [3]. Для иллюстрации сказанного на рис. 1 представлены результаты расчетов изменения во времени скорости необратимого распространения неустойчивости вдоль сверхпроводящего композита, охлаждаемого жидким гелием. Для общности получаемых результатов расчеты зависимостей $v(t)$ проводились на основе анализа кинетики точек с температурами $T_{c s}$ и $T_{0}+1$, а также точки с напряженностью $E_{c}$. На рис. $1, a$ изображено изменение во времени скорости распространения $T_{c s}$ (сплошная линия) и $T_{0}+1$ (штриховая линия) как для докритических, так и для закритических токов. При этом для тока $I=500$ А вычисления проводились для возмущений с различной начальной температурой. Они подтверждают независимость установившегося значения скорости распространения тепловой неустойчивости от энергии закритического возмущения после формирования волны переключения. На рис. 1, $b$ приведены кривые, описывающие изменение во времени скорости распространения изотермы $T_{c s}$ (сплошная линия) и точки с напряженностью $E_{c}$ (штриховая линия). Результаты 

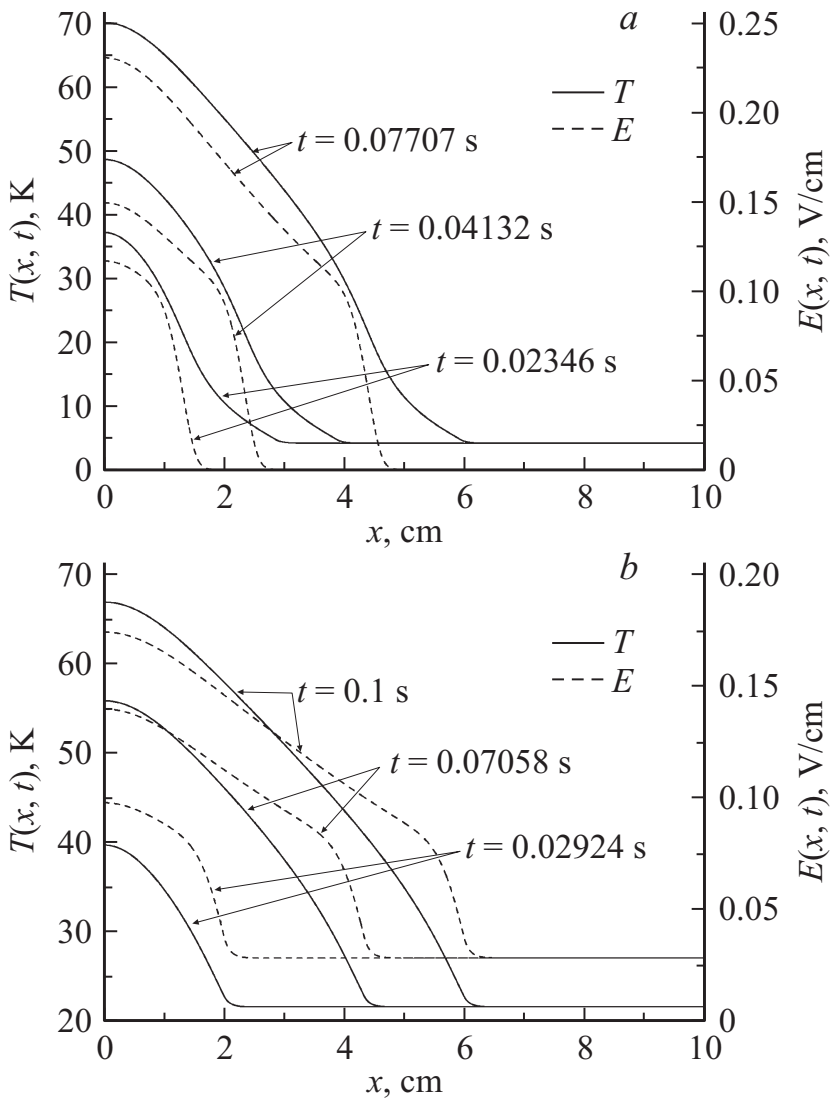

Рис. 3. Образование теплоэлектрической волны переключения в результате действия локального возмущения и охлаждения композита жидким гелием $\left(a-I=500 \mathrm{~A}, x_{1}=0.5 \mathrm{~cm}\right.$, $\left.T_{1}=20 \mathrm{~K}\right)$ и жидким водородом $\left(b-I=400 \mathrm{~A}, x_{1}=0.5 \mathrm{~cm}\right.$, $\left.T_{1}=25 \mathrm{~K}\right)$.

выполненных расчетов показывают, что для определения скорости волны переключения все приближения эквиваленты.

Рис. 2 демонстрирует кинетику изотермы $T_{c s}$ в случае интенсивного и неинтенсивного охлаждения композита при $T_{0}=4.2 \mathrm{~K}$ и условиях (6), (8). Сопоставляя между собой результаты, представленные на рис. 2, нетрудно заметить, что при докритических токах установившиеся значения скоростей распространения тепловых неустойчивостей несущественно отличаются друг от друга даже при столь значительном отличии в интенсивности режимов охлаждения. Отсюда следует важный вывод о том, что жидкий гелий не оказывает эффективного стабилизирующего влияния на необратимое распространение тепловой неустойчивости вдоль композита.

На рис. 3, $а$ представлены кривые, демонстрирующие особенности формирования теплоэлектрических волн переключения в композите с нелинейной зависимостью удельного электрического поля от температуры при его охлаждении жидким гелием. Видно, что температура и напряженность электрического поля в центре возмущения постоянно возрастают. Это означает, что температура теплового равновесия композита в наиболее нагретой части при его переходе в нормальное состояние отсутствует. Как следует из рис. 2, это объясняется тем, что в этом случае тепловыделение существенно превышает теплоотдачу в хладагент, и тепловое состояние композита в наиболее нагретой части приближается к адиабатическому. Эта закономерность объясняет возможность пережога интенсивно охлаждаемого композита, если из него не начать во время выводить ток. Подобные состояния возникают и при охлаждении композита жидким водородом (рис. $3, b$ ). Но в этом случае по вполне понятным причинам нарастание температуры композита не столь интенсивно.

Таким образом, в нелинейном приближении по температуре возникновение и развитие тепловых неустойчивостей в технических сверхпроводниках имеет ряд специфических особенностей, которые необходимо учитывать как при теоретическом, так и экспериментальном анализе условий их тепловой стабилизации. В результате при определении скоростей распространения тепловых неустойчивостей будут иметь место следующие закономерности.

На рис. 4 представлены зависимости от тока скорости установившегося распространения тепловых неустойчивостей вдоль композита, охлаждаемого жидким гелием, а на рис. 5 для того же композита, но охлаждаемого жидким водородом. В обоих случаях расчеты выполнялись как для идеальной ВАХ при $0<I<I_{c}$, так и для степенной $\mathrm{BAX}$ при докритических и закритических токах $\left(0<I<I_{q}\right)$. Для рассматриваемого композита, охлаждаемого жидким гелием или жидким водородом, значения токов срыва равны $I_{q}=1019$ А и $I_{q}=608 \mathrm{~A}$ соответственно, как следует из расчетов, приведенных в Приложении. На вставках к каждому из рисунков соответствующие зависимости показаны более детально

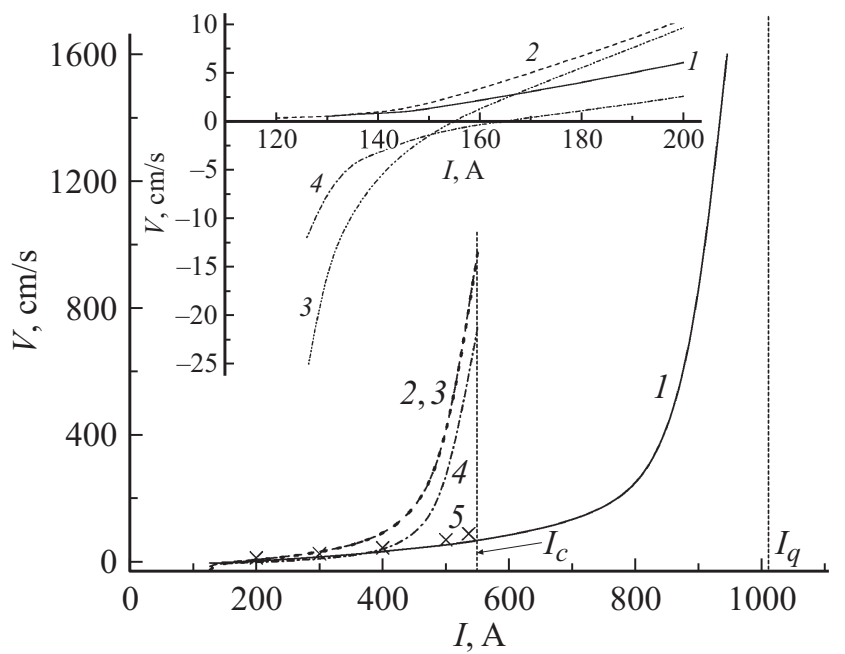

Рис. 4. Скорость распространения теплового возмущения как функция тока при охлаждении композита жидким гелием (1 расчет по модели (1), (2), (4)-(6); 2, 3, 4- расчет модели $(1)-(3),(6))$ и при неинтенсивном охлаждении $(5-$ расчет по модели (1), (2), (4), (5), (8)). 


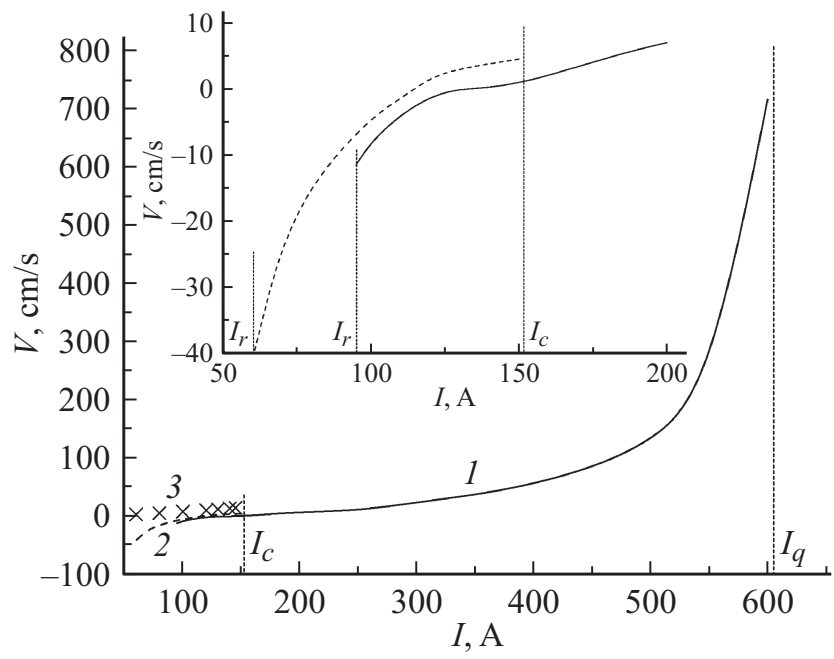

Рис. 5. Скорость волны переключения как функция тока при охлаждении композита жидким водородом (1 - расчет по моделям (1), (2), (4), (5), (7); 2 - расчет модели (1)-(3), (7)), и при неинтенсивном охлаждении при $T_{0}=20 \mathrm{~K}(3-$ расчет по моделям (1), (2), (4), (5), (8)).

в области малых токов. Отметим, что определение скоростей распространения неустойчивостей в композите, охлаждаемом жидким гелием, при токах, меньших 100 А, приводит к значительному увеличению времени расчета, поскольку при малых токах существенно возрастает время формирования волны переключения. Поэтому моделирование процессов распространения тепловой неустойчивости для данных токовых режимов не выполнялось. Тем не менее укажем, что кривые 1,2 имеют общее начало при $I=0$, так как расчеты, выполненные согласно теореме равных площадей, показывают, что минимальный ток распространения нормальной зоны $I_{s}$, а значит, и минимальный ток существования нормальной зоны $I_{r}$, равны нулю, даже несмотря на использование в качестве хладагента жидкого гелия. Другими словами, в данном случае отсутствует область стационарной стабилизации.

Чтобы продемонстрировать влияние нелинейного характера нарастания с температурой удельного электрического сопротивления матрицы и коэффициента ее теплопроводности на характер изменения скорости распространения неустойчивости, на рис. 4 приведены результаты расчетов $v(I)$, выполненных для различных температурных зависимостей $\rho_{m}$ и $\lambda$. Кривые 1 и 2 соответствуют расчету по моделям (1), (2), (4)-(6) и $(1)-(3),(6)$ соответственно, в которых учитывались температурные зависимости $\rho_{m}$ и $\lambda$. Кривая 3 рассчитана при $\rho_{m}(4.2 \mathrm{~K})$, а кривая $4-$ при условии, что значения $\rho_{m}$ и $\lambda$ не зависят от температуры и принимались равными $\rho_{m}(4.2 \mathrm{~K})$ и $\lambda(4.2 \mathrm{~K})$. В этих случаях численные эксперименты выполнялись в рамках модели (1)-(3), (6).
Представленные результаты наглядно показывают, что скорости распространения тепловых неустойчивостей в сверхпроводящем композите с серебряной матрицей, охлаждаемого жидким гелием, оказываются неотрицательными во всем диапазоне устойчивого ввода транспортного тока (кривые 1, 2 на рис. 4). В основе данной закономерности, как отмечалось выше, лежит интенсивное нарастание с температурой мощности джоулева тепловыделения по сравнению с температурным ростом мощности теплового потока, отводимого в жидкий гелий при пленочном кипении. В результате разогрев композита в области возникновения неустойчивости приближается к адиабатическому. При этом значения $v(I)$ для интенсивно охлаждаемого композита со степенной ВАХ (кривая 1 на рис. 4) в диапазоне токов от нуля до $I_{c}$ по своему характеру нарастания с током близки к соответствующим значениям скоростей тепловых неустойчивостей при неинтенсивном охлаждении композита. Более того, как отмечалось выше, значение $I_{s}$ в рассматриваемом случае равно нулю, что также характерно для неохлаждаемых сверхпроводящих композитов. Следовательно, вывод об адиабатическом характере процессов, происходящих в охлаждаемом жидким гелием композите в области его наибольшего разогрева, справедлив вплоть до априори заданного значения его критического тока. Другими словами, в широком диапазоне изменения тока жидкий гелий не позволяет обеспечить эффективную тепловую стабилизацию композитов на основе высокотемпературных сверхпроводников. В этом случае их пережог оказывается весьма вероятным, что хорошо известно из практики их применения.

Следует также отметить, что отличие между моделями с идеальной и со степенной ВАХ возрастает с увеличением тока и становится весьма заметным (рис. 4) при токах, близких к критическому току композита, так как при учете реальной BAX токовые неустойчивости могут не возникать при токах, значительно превышающих условно заданное значение $I_{c}$.

При использовании в качестве хладагента жидкого водорода наблюдается заметное отличие в значениях минимальных токов существования нормальной зоны $I_{r}$ (рис. 5), определенных в рамках обеих моделей ВАХ, в силу соответствующего отличия в расчетных зависимостях критической плотности тока от температуры. Кроме этого, существенно отличаются и значения токов, устойчиво протекающих по композиту. Как следствие, существует значительный диапазон закритических токов, в котором переход в нормальное состояние происходит не скачкообразно, а в виде волны переключения, распространяющейся с высокой скоростью. В то же время расчетные значения скорости волны переключения, определенные в рамках обеих моделей ВАХ в диапазоне токов $I_{s}<I<I_{c}$, малы и незначительно отличаются друг от друга. Это указывает на эффективное охлаждение композита жидким водородом при малых токах. Однако при закритических токах будут возникать состояния, близкие к адиабатическим (рис. 5). 


\section{Условие лавинообразного саморазогрева технического сверхпроводника}

Как следует из обсужденных выше результатов численных экспериментов, переход технического сверхпроводника в нормальное состояние может не сопровождаться катастрофически быстрым нарастанием его температуры как при использовании в качестве хладагента жидкого гелия, так и жидкого водорода. На рис. 6,a построены кривые, описывающие изменение во времени температуры композита, охлаждаемой жидким гелием, в его наиболее разогретой части после действия локального температурного возмущения $\left(x_{1}=0.5 \mathrm{~cm}\right) \mathrm{c}$ различными значениями температур начального нагрева композита. Расчеты проводились как для докритических, так и для закритических токов. В первом случае они выполнялись на основе моделей (1)-(6), а во втором по моделям (1), (2), (4)-(6). Представленные результаты показывают, что переход композита в нормальное состояние при докритических токах (кривые $3,3^{\prime}, 4,4^{\prime}$ ) не характеризуется интенсивным ростом его температуры. Поэтому существует время, позволяющее избежать пережог композита, выводя из него ток. К этому выводу приводят расчеты по обеим моделям ВАХ. Однако вероятность быстрого пережога композита повышается, когда по нему будут течь закритические токи, особенно близкие к току срыва (кривая 5). В то же время при использовании в качестве хладагента жидкого водорода нагрев композита на основе $\mathrm{Bi}_{2} \mathrm{Sr}_{2} \mathrm{CaCu}_{2} \mathrm{O}_{8}$ даже при закритических токах не столь интенсивный. Для данных режимов существуют приемлемые времена вывода тока, позволяющие избежать пережог. Это следует из рис. $6, b$, где приведены кривые, описывающие изменение во времени температуры в наиболее нагретой точке при

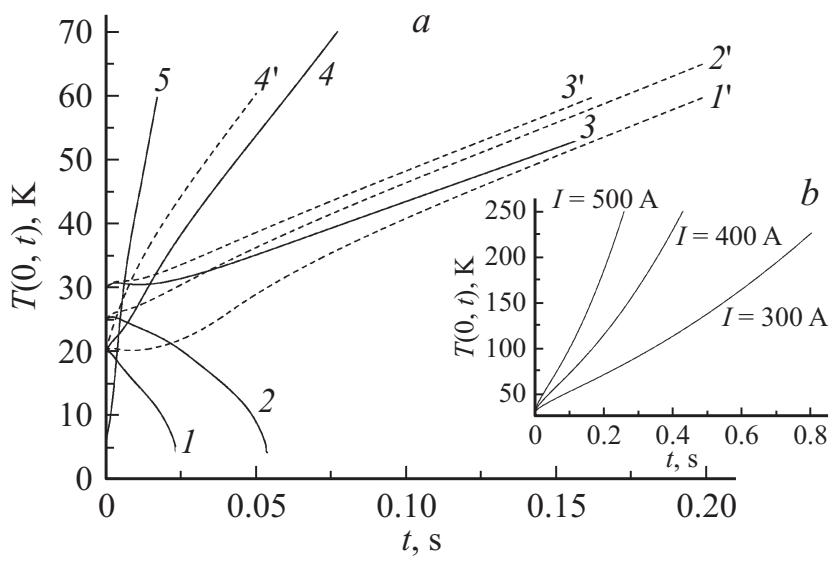

Рис. 6. Изменение температуры в центре сверхпроводящего композита, охлаждаемого жидким гелием (a) (сплошная линия - модель ВАХ (4), штриховая линия - модель ВАХ (3): $1,1^{\prime}-I=300 \mathrm{~A}, T_{1}=20 \mathrm{~K} ; 2,2^{\prime}-I=300 \mathrm{~A}, T_{1}=25 \mathrm{~K} ; 3$, $3^{\prime}-I=300 \mathrm{~A}, T_{1}=30 \mathrm{~K} ; 4,4^{\prime}-I=500 \mathrm{~A}, T_{1}=20 \mathrm{~K} ; 5-$ $\left.I=900 \mathrm{~A}, T_{1}=6 \mathrm{~K}\right)$ и жидким водородом $(b)$.

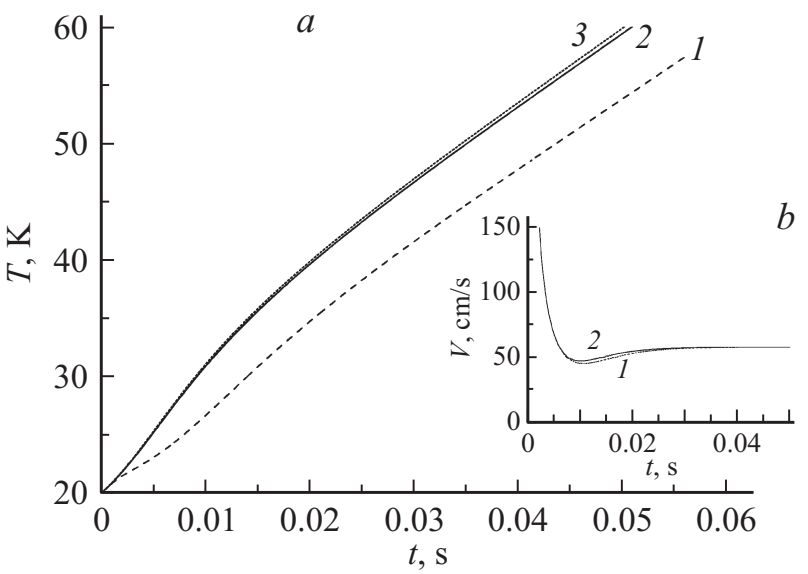

Рис. 7. Нарастание температуры в центре сверхпроводящего композита $(a)$ и изменение во времени скорости тепловой неустойчивости $(b)$ при его охлаждении жидким гелием и действии возмущения с различной начальной длиной: 1 $x_{1}=0.5 \mathrm{~cm} ; 2-x_{1}=8 \mathrm{~cm} ; 3-$ приближение в рамках модели однородного состояния (9).

действии локального возмущения $x_{1}=1 \mathrm{~cm}, T_{1}=30 \mathrm{~K}$ и закритических токах.

Результаты, представленные на рис. 6, $a$, также приводят к выводу о том, что расчет критических энергий возникновения тепловых неустойчивостей в сверхпроводящем композите при его интенсивном охлаждении будет зависеть от выбора расчетной модели ВАХ. Действительно, согласно модели с идеальной ВАХ, локальное температурное возмущение с начальной температурой разогрева $T_{1}=20 \mathrm{~K}$ при токе $I=300 \mathrm{~A}$ переводит композит в нормальное состояние (кривая $1^{\prime}$ ). В то же время расчет теплового состояния композита, выполненный в рамках модели со степенной ВАХ, показывает, что композит сохраняет сверхпроводящие свойства даже при ее локальном нагреве до температуры $T_{1}=25 \mathrm{~K}$ (кривая 2), т.е. при существенно большем перегреве. Отметим, что это отличие будет наблюдаться и при действии возмущений произвольной протяженности.

Для оценки температуры максимального разогрева технических сверхпроводников можно воспользоваться более упрощенной моделью, пренебрегая механизмом теплопроводности. На рис. 7, $a$ между собой сопоставлены результаты расчета изменения во времени температуры сверхпроводящего композита с током $I=500$ А, охлаждаемого жидким гелием, при действии температурного возмущения $T_{1}=20 \mathrm{~K}$ различной протяженности. Соответственно кривая 1 описывает разогрев композита при локальном возмущении, кривая 2 - при протяженном возмущении. Расчет кривой 3 основывался на численном решении уравнения теплового баланса

$$
C(T) \frac{d T}{d t}=E J-\frac{q(T) p}{S}, \quad T(0)=T_{1},
$$

в котором напряженность электрического поля определялась согласно (4), а критическая плотность тока опи- 
сывалась зависимостью (5). Решение данного уравнения может быть выписано в квадратурах

$$
\int_{T_{1}}^{T} \frac{C(T) d T}{E J-q(T) p / S}=t .
$$

Уравнение (9) описывает однородные тепловые состояния композита, которые возникают при бесконечно протяженном начальном температурном возмущении $\left(x_{1} \rightarrow \infty\right)$. Поэтому результаты расчета температуры разогрева сверхпроводящей композита, основанные на решении начально-краевой задачи $(1),(2)$, будут всегда приводить к более оптимистичным оценкам условий его пережога, критических значений температуры начального возмущения по сравнению с оценками, к которым приводит уравнение (9). В то же время правомерность его использования для расчета температуры технических сверхпроводников приводит к выводу о том, что пережог, т.е. температура в наиболее нагретой точке, не зависит от скорости распространения тепловой неустойчивости вдоль сверхпроводника. Последняя влияет только на размер области пережога (рис. 7,b). Для верхней оценки температуры и времени пережога можно воспользоваться решением (10), как это демонстрирует рис. $7, a$.

Из (9) и (10) также следует, что известная модель „hot spot“ позволяет выполнить анализ пережога сверхпроводящих композита при действии бесконечно протяженного возмущения для наиболее неблагоприятных условий отвода выделяемого джоулева тепла, когда в уравнении (1) не учитывается и теплопроводность, и теплоотдача в хладагент. Этот вывод демонстрирует ошибочность широко распространенного мнения о том, что модель ,hot spot“" описывает нагрев технического сверхпроводника, когда вся запасенная энергия выделяется в точке возникновения неустойчивости [15].

Уравнение (9) позволяет также дать простое объяснение причины возникновения условий катастрофического нарастания температуры у технических сверхпроводников при необратимом распространении тепловой неустойчивости. Для этого воспользуемся моделью с идеальной BAX и перепишем правую часть уравнения (9), принимая во внимание особенности изменения с температурой мощности джоулевого тепловыделения и потока тепла, отводимого в хладагент. Во-первых, как следует из $[11,12]$, удельное электрическое сопротивление серебра при его нагреве до температуры порядка $T_{r}=20 \mathrm{~K}$ слабо зависит от температуры, но при $T_{1}>T_{r}$ возрастает практически линейно. Поэтому его значение при $T_{1}>T_{r}$ может быть записано в упрощенном виде: $\rho_{m}(T)=\rho_{r}+\left(T-T_{r}\right) d \rho_{m} / d T$. Здесь $\rho_{r}-$ остаточное сопротивление, $d \rho_{m} / d T=$ const - скорость температурного нарастания удельного электрического сопротивления, $T_{r}$ - температура, после которой начинается рост сопротивления матрицы. Во-вторых, согласно (6) и (7), тепловой поток в жидкий хладагент при высоких перегревах, т.е. при пленочном кипении, с удовлетворительной степенью точности может быть аппроксимирован зависимостью вида $q(T)=h\left(T-T_{0}\right)$, где $h-$ эффективное значение коэффициента теплоотдачи. В результате тепловое состояние технического сверхпроводника при $T>T_{c B}$, согласно (3), (9), может быть записано как

$$
\begin{aligned}
C(T) & \frac{d T}{d t}= \\
& \left\{\begin{array}{l}
\frac{J^{2} \rho_{r}}{1-\eta}-\frac{h p}{S}\left(T-T_{0}\right), T_{c B} \leq T \leq T_{r}, \\
\frac{J^{2}}{1-\eta}\left[\rho_{r}+\frac{d \rho_{m}}{d T}\left(T-T_{r}\right)\right]-\frac{h p}{S}\left(T-T_{0}\right), T>T_{r} .
\end{array}\right.
\end{aligned}
$$

Перепишем его в виде

$$
\begin{aligned}
& C(T) \frac{d T}{d t}= \\
& \left\{\begin{array}{c}
-\frac{h p}{S} T+q_{1}, q_{1}=\frac{J^{2} \rho_{r}}{1-\eta}+\frac{h p}{S} T_{0}, T_{c B} \leq T \leq T_{r}, \\
\left(\frac{J^{2}}{1-\eta} \frac{d \rho_{m}}{d T}-\frac{h p}{S}\right) T+q_{2}, \\
q_{2}=\frac{J^{2}}{1-\eta}\left[\rho_{r}-\frac{d \rho_{m}}{d T} T_{r}\right]+\frac{h p}{S} T_{0}, T>T_{r} .
\end{array}\right.
\end{aligned}
$$

Уравнение (11) при $T>T_{r}$ относится к классу уравнений, описывающих диффузионные явления в активной среде с размножением, которые при определенных условиях носят цепной характер взрывного типа. Например, по аналогии с процессом деления тяжелых ядер [16] значение $T$ описывает концентрацию нейтронов. Тогда $\gamma_{1}=\frac{J^{2}}{(1-\eta)} \frac{d \rho_{m}}{d T}-$ коэффициент их рождения, $\gamma_{2}=\frac{h p}{S}-$ коэффициент поглощения, $q_{2}$ - мощность объемного источника, который в зависимости от знака $q_{2}$ описывает либо генерацию $\left(q_{2}>0\right)$, либо поглощение $\left(q_{2}<0\right)$ нейтронов. Соответственно если $\gamma=\gamma_{1}-\gamma_{2}>0$, то процесс генерации нейтронов будет преобладать над их поглощением. В этом случае увеличение $T$ будет иметь лавинообразной характер. В частности, в простейшем случае, когда $C=$ const, решение уравнения (11) при $T>T_{r}$ записывается в виде

$$
T=\left(T_{r}+\frac{q_{2}}{\gamma}\right) \exp \left(\frac{\gamma}{C} t\right)-\frac{q_{2}}{\gamma},
$$

т.е. при саморазогреве технического сверхпроводника может возникнуть экспоненциальное нарастание его температуры (рис. 1-3). Этот процесс зависит от значений $J, d \rho_{m} / d T, \rho_{r}, C, h, \eta, p / S$. В частности, он тем вероятнее, чем больше величина $\frac{J^{2}}{(1-\eta)} \frac{d \rho_{m}}{d T}$. Но при этом стабилизирующее действие на не контролируемый рост температуры будут оказывать коэффициент теплоотдачи и теплоемкость. Как следствие, лавинообразное нарастание температуры композита неизбежно при условиях охлаждения, близких к адиабатическим. Именно при этих условиях будет находиться технический сверхпроводник, охлаждаемый жидким гелием, когда его наиболее нагретая часть в результате саморазогрева 


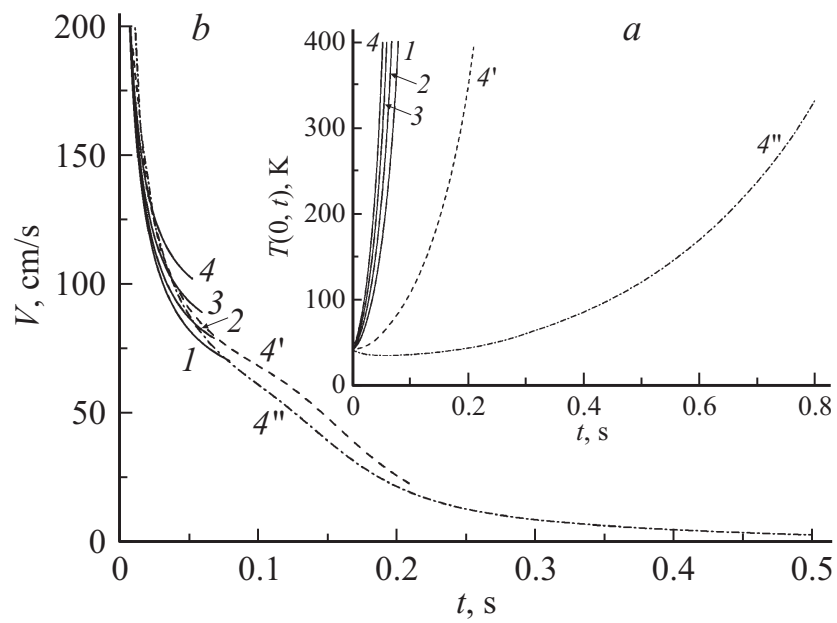

Pис. 8. Изменение во времени температуры $\mathrm{YBa}_{2} \mathrm{Cu}_{3} \mathrm{O}_{7}$ ленты $(a)$ и скорости тепловой неустойчивости $(b)$ в результате действия закритического локального возмущения $\left(x_{1}=1 \mathrm{~cm}, T_{1}=40 \mathrm{~K}\right)$ при $T_{0}=15 \mathrm{~K}: 1-I=160,2-170$, $3-180,4,4^{\prime}, 4^{\prime \prime}-190 \mathrm{~A} ;(1-4)-a_{\mathrm{cu}}=45 \mu \mathrm{m}, 4^{\prime}-$ $a_{\mathrm{cu}}=100 \mu \mathrm{m}, 4^{\prime \prime}-a_{\mathrm{cu}}=200 \mu \mathrm{m}$.

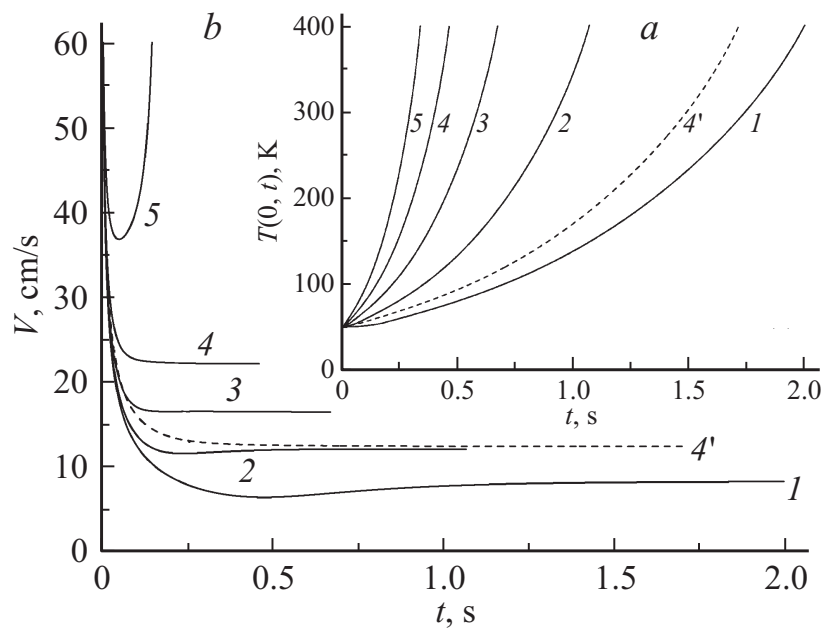

Pис. 9. Изменение во времени температуры $\mathrm{YBa}_{2} \mathrm{Cu}_{3} \mathrm{O}_{7}$ ленты $(a)$ и скорости тепловой неустойчивости $(b)$ в результате действия закритического протяженного возмущения $\left(x_{1}=10 \mathrm{~cm}, T_{1}=50 \mathrm{~K}\right)$ при $T_{0}=40 \mathrm{~K}: 1-I=30,2-$ $40,3-50,4,4^{\prime}-60 \mathrm{~A} ;(1-4)-a_{\mathrm{cu}}=45 \mu \mathrm{m}, 4^{\prime}-$ $a_{\mathrm{cu}}=100 \mu \mathrm{m}$.

находится в режиме пленочного кипения. Подчеркнем, что обсужденные особенности возникновения пережога технического сверхпроводника описывают обе модели $\mathrm{BAX}$, и они наглядно демонстрируют, что в основе его пережога лежит тривиальный рост с температурой сопротивления стабилизирующего покрытия, приводящий к нарушению условий теплового равновесия технического сверхпроводника, и прежде всего несущего ток высокой плотности.

Обсужденные характерные закономерности формирования неустойчивых тепловых состояний
$\mathrm{Ag} / \mathrm{Bi}_{2} \mathrm{Sr}_{2} \mathrm{CaCu}_{2} \mathrm{O}_{8}$, сформулированные выше, соблюдаются и при анализе условий тепловой стабилизации сверхпроводящих лент на основе $\mathrm{YBa}_{2} \mathrm{Cu}_{3} \mathrm{O}_{7}$ (Y123). В частности, на рис. 8, 9 представлены результаты численного моделирования кинетики распространения тепловой неустойчивости в неинтенсивно охлаждаемой ленте Y123 с двумя стабилизирующими покрытиями из серебра и меди, описанной в [17]. Соответственно ее геометрические параметры (ширина $b$, толщина сверхпроводника $a_{s}$, толщина серебра $a_{\text {ag }}$ и толщина меди $\left.a_{\mathrm{cu}}\right)$ задавались равными $b=0.2 \mathrm{~cm}, a_{s}=10^{-4} \mathrm{~cm}, a_{\mathrm{ag}}=$ $=17 \cdot 10^{-4} \mathrm{~cm}, \quad a_{\mathrm{cu}}=45 \cdot 10^{-4} \mathrm{~cm}, \quad$ а плотность критического тока сверхпроводника была описана линейной зависимостью вида $J_{c}=J_{c 0}\left(T_{c B}-T\right) /$ $/\left(T_{c B}-T_{0}\right) \quad$ с критическими параметрами $J_{c 0}=$ $=6.04 \cdot 10^{9} \mathrm{~A} / \mathrm{cm}^{2}$ и $T_{c B}=55 \mathrm{~K}$ во внешнем магнитном поле $B=10 \mathrm{~T}$ и $T_{0}=15 \mathrm{~K}$. Параметры ВАХ задавались равными $E_{c}=10^{-6} \mathrm{~V} / \mathrm{cm}, n=22$. Для расчета объемной теплоемкости и удельного электрического сопротивления меди использовались результаты $[11,12,18]$, принимая $\rho_{\mathrm{cu}}(273 \mathrm{~K})=1.55 \cdot 10^{-6} \Omega \cdot \mathrm{cm}$ и $\mathrm{RRR}=100$. В уравнении (1) тепловыделение внутри ленты $G=E J$ рассчитывалось по формуле

$$
G(T)= \begin{cases}\eta J_{c 0} E \frac{T_{c B}-T}{T_{c B}-T_{0}}\left(\frac{E}{E_{c}}\right)^{1 / n}+\frac{E^{2}}{\rho_{k}}, & T<T_{c B}, \\ \frac{E^{2}}{\rho_{k}}, & T \geq T_{c B},\end{cases}
$$

где

$$
\rho_{k}(T, B)=\frac{\left(a_{s}+a_{\mathrm{ag}}+a_{\mathrm{cu}}\right) \rho_{\mathrm{ag}}(T, B) \rho_{\mathrm{cu}}(T, B)}{a_{\mathrm{cu}} \rho_{\mathrm{ag}}(T, B)+a_{\mathrm{ag}} \rho_{\mathrm{cu}}(T, B)} .
$$

Для расчета теплового потока в хладагент использовалась формула (8) при $h=10^{-3} \mathrm{~W} /\left(\mathrm{cm}^{2} \cdot \mathrm{K}\right)$, в которой температура хладагента задавалась равной $T_{0}=$ $=15 \mathrm{~K}$ или $T_{0}=40 \mathrm{~K}$.

Как следует из сформулированного условия возникновения пережога технического сверхпроводника и наглядно демонстрируют рис. 8, 9, лавинообразный процесс саморазогрева ленты Ү123 может развиваться без образования теплоэлектрической волны переключения (прежде всего в силу высокой плотности введенного тока). В этом случае протекающие процессы и в том числе распространение тепловой неустойчивости имеют существенно нестационарный характер, в результате которого может произойти быстрый пережог ленты. С другой стороны, увеличение толщины медного покрытия или увеличение температуры хладагента будет приводит к улучшению условий тепловой стабилизации ленты Y123, так как при этом уменьшается плотность транспортного тока. Как следствие, интенсивность нарастания температуры ленты уменьшается, позволяя принять меры по предотвращению ее пережога.

\section{Выводы}

Анализ скоростей необратимого распространения тепловой неустойчивости вдоль технического сверхпро- 
водника показал существование особых условий его тепловой стабилизации, если учитывать нелинейные температурные зависимости свойств сверхпроводника и стабилизирующей матрицы. Установлено, что у интенсивно охлаждаемых токонесущих элементов сверхпроводящих магнитных систем могут отсутствовать минимальные токи существования и распространения нормальной зоны, определяющие диапазон токов, в котором сверхпроводящее состояние устойчиво к произвольным тепловым возмущениям. Данная особенность условий тепловой стабилизации является следствием нелинейной температурной зависимости удельного электрического сопротивления стабилизирующей матрицы, когда при температурах выше критической температуры сверхпроводника нарастание мощности джоулева тепловыделения превышает температурный рост мощности теплового потока в хладагент. Как следствие, при этих режимах отсутствует область стационарной стабилизации, а установившиеся значения скоростей распространения тепловых неустойчивостей во всем диапазоне изменения токов не принимают отрицательных значений.

Показано, что развитие тепловой неустойчивости в техническом сверхпроводнике может как приводить, так и не приводить к образованию теплоэлектрической волны переключения. В первом случае нарушение тепловой стабильности после возникновения волны переключения, распространяющейся вдоль технического сверхпроводника с постоянной скоростью, происходит не только при докритических токах, но и в области закритических токов, не превышающих предельно допустимое значение. Разрушение сверхпроводимости без образования теплоэлектрической волны переключения характерно для технических сверхпроводников с высокими значениями плотности транспортного тока. В целом для корректного расчета кинетики теплового возмущения во внимание необходимо принимать нелинейный характер изменения с температурой свойств сверхпроводника и стабилизирующей матрицы.

Доказано, что развитие тепловой неустойчивости в техническом сверхпроводнике имеет характер цепной реакции взрывного типа. Выписано условие ее возникновения. Из него следует, что даже при интенсивных условиях охлаждения вероятность пережога тем выше, чем выше плотность тока в сверхпроводнике и чем быстрее нарастает с температурой удельное электрическое сопротивление матрицы.

\section{Приложение}

Будем искать предельно допустимый ток, устойчиво протекающий по сверхпроводящему композиту, в соответствии с итерационной процедурой, в основе которой лежит метод конечного возмущения исходного равновесного состояния, предложенный в [19]. А именно на основе численного решения уравнения (1) с видоизмененным начальным условием $T(x, 0)=T_{0}$ необходимо

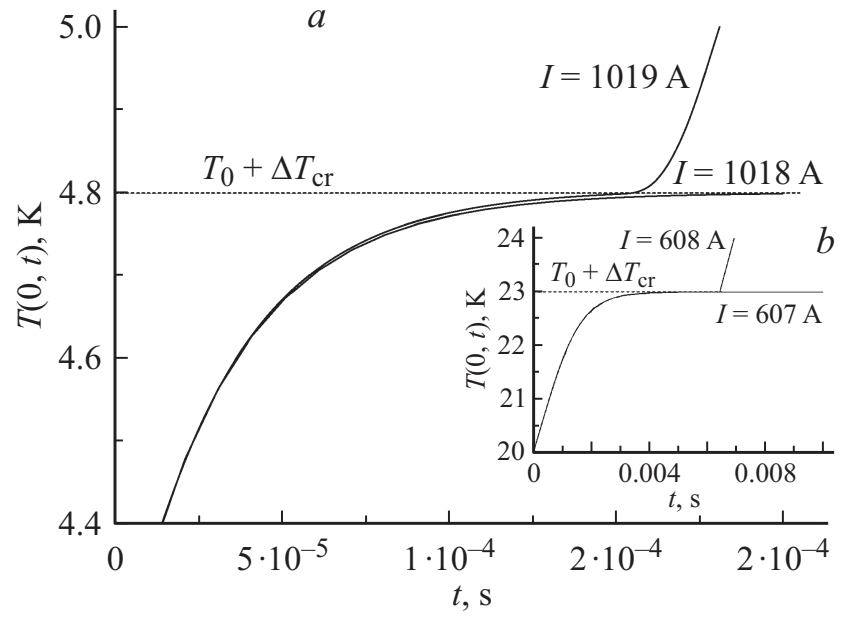

Рис. 10. Изменение температуры $\mathrm{Ag} / \mathrm{Bi}_{2} \mathrm{Sr}_{2} \mathrm{CaCu}_{2} \mathrm{O}_{8}$, охлаждаемого жидким гелием (a) или жидким водородом $(b)$, при вводе токов, близких к предельно допустимым.

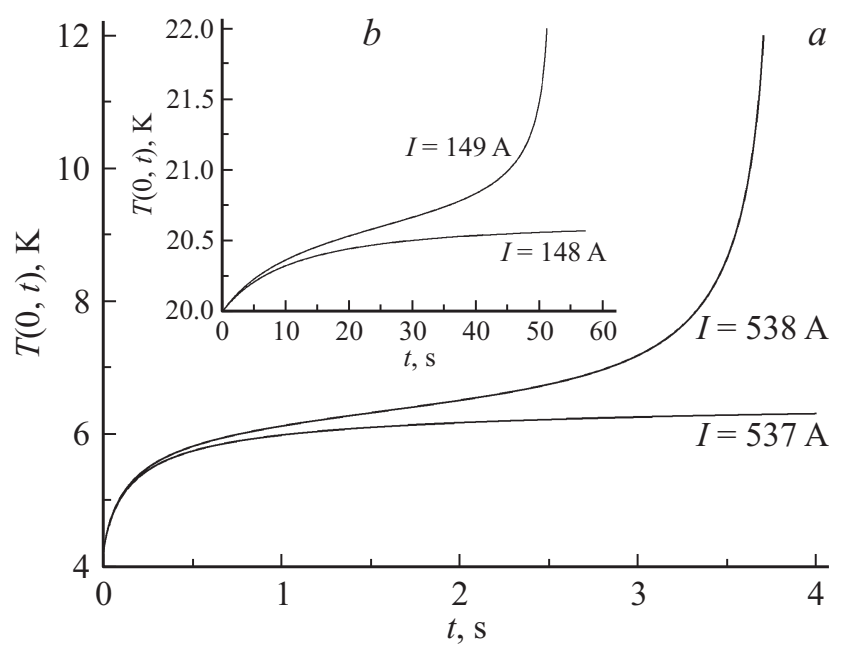

Рис. 11. Токи возникновения неустойчивости при неинтенсивном охлаждении $\mathrm{Ag} / \mathrm{Bi}_{2} \mathrm{Sr}_{2} \mathrm{CaCu}_{2} \mathrm{O}_{8}: a-T_{0}=4.2 \mathrm{~K}, b-$ $T_{0}=20 \mathrm{~K}$.

определить два таких значения тока, при вводе которых происходит либо стабилизация, либо необратимый рост температуры композита. Соответственно в первом случае введенный ток устойчив, а во втором - приводит к спонтанному переходу композита в нормальное состояние. На рис. 10, 11 приведены результаты численных расчетов токов срыва для $\mathrm{Ag} / \mathrm{Bi}_{2} \mathrm{Sr}_{2} \mathrm{CaCu}_{2} \mathrm{O}_{8}$ при условиях охлаждения (6)-(8). Как следует из расчетов, ток срыва при охлаждении композита жидким гелием равен 1019 А (рис. 10,a), а при охлаждении композита жидким водородом - 608 А (рис. $10, b)$. Они существенно превышают соответствующие значения критических токов рассматриваемого композита. При этом токовые неустойчивости в обоих случаях возникают в силу перехода от режима пузырькового кипения хладагента к пленочному. Кривые, приведенные на рис. 11, описывают изменение 
во времени температуры в центре ленты в случае ее неинтенсивного охлаждения при вводе токов, близких к токам срыва. В этом случае теплоотдача в хладагент описывалась уравнением (8) при $T_{0}=4.2 \mathrm{~K}$ и $T_{0}=20 \mathrm{~K}$. Для данных режимов охлаждения токи возникновения тепловой неустойчивости меньше соответствующих значений критических токов.

Таким образом, в зависимости от условий охлаждения технического сверхпроводника диапазон токов, в котором соблюдается его тепловая стабильность, может быть как докритическим, так и закритическим. Это необходимо учитывать при экспериментальном исследовании условий тепловой стабильности технических сверхпроводников с реальными ВАХ.

\section{Список литературы}

[1] Альтов В.А., Зенкевич В.Б., Кремлев М.Г., Сычев В.В. Стабилизация сверхпроводящих магнитных систем. М.: Энергоатомиздат, 1984. $312 \mathrm{c}$.

[2] Уилсон М. Сверхпроводящие магниты. М.: Мир, 1985. $407 \mathrm{c}$.

[3] Гуревич А.Вл., Мини Р.Г., Рахманов А.Л. Физика композитных сверхпроводников. М.: Наука, 1987. 240 с.

[4] Клименко Е.Ю., Мартовецкий Н.Н., Новиков С.И. В кн.: Техническая сверхпроводимость в электроэнергетике и электротехнике. М. СЭВ, 1986. С. 161-187.

[5] Paasi J., Lehtonen J., Kalliohaka T. et al. // Supercond. Sci. Technol. 2000. Vol. 13. P. 949-954.

[6] Majoros A., Campbell A.M., Glowacki B.A. et al. // Physica C. 2004. Vol. 401. P. 140-145.

[7] Ishiyama A., Yanai M., Morisaki T. et al. // IEEE Trans. Appl. Supercon. 2005. Vol. 15. N 2. P. 1659-1662.

[8] Рахманов А.Л., Иванов С.С., Баламов Н.Н. и др. // Прикладная физика и математика. 2013. № 1. С. 68-73.

[9] Romanovskii V.R., Watanabe K., Awaji S., Nishïima G. // Supercond. Sci. Tech. 2006. Vol. 19. N 8. P. 703-710.

[10] Bottura L. Critical Surface for BSCCO-2212 Superconductor, Note-CRYO/02/027, CryoSoft library, CERN, 2002.

[11] Dresner L. // Cryogenics. 1993. Vol. 33. N 9. P. 900-909.

[12] Lim H., Iwasa Y. // Cryogenics. 1997. Vol. 37. N 12. P. 789 799.

[13] Herrmann P.F., Albrecht C., Bock J. et al. // IEEE Trans. Appl. Supercon. 1993. Vol. 3. N 1. P. 876-880.

[14] Brentari E.G., Smith R. // Adv. Cryog. Eng. 1965. Vol. 10. P. 325-341.

[15] Iwasa Y. Case Studies in Superconducting Magnets. 2nd Edition. Springer Science. 2009. 661 p.

[16] Яворский Б.М., Детлаф А.А. Справочник по физике. М.: Наука, 1968. 939 с.

[17] Awaji S., Hou Y., Oguro H., Watanabe K. et al. // IEEE Trans Appl Supercon. 2012. Vol. 22. N 3. P. 6601004.

[18] Seeber B. Handbook of Applied Superconductivity. Editor B. Seeber. Bristol: IOP Publishing, 1998. Vol. 1. P. 1067-1082.

[19] Кейлин В.Е., Романовский В.Р. // ЖТФ. 1993. Т. 63. Вып. 1. C. $10-21$. 